\title{
Generalidades y tratamiento de la Sarcopenia
}

\author{
José Hernández-Rodríguez* \\ Manuel Emiliano Licea-Puig*
}

\begin{abstract}
*Médico Endocrinólogo. Profesor Auxiliar. Investigador del Grupo Diabetes Mellitus del Instituto Nacional de Endocrinología del Ministerio de Salud Pública de Cuba. La Habana. Cuba.

Correspondencia: Dr. José Hernández Rodríguez. Dirección: Avenida 17 esquina a D \# 509. Vedado. Habana. CP. 10400. Cuba. Teléfono: 83275 77-79; 832 3545, Extensión 121. Correo electrónico: pepehdez@infomed.sld.cu
\end{abstract}

\section{RESUMEN}

Introducción: la sarcopenia hace referencia a la disminución de la masa y fuerza muscular relacionada con los mecanismos del envejecimiento y genera un compromiso funcional de alto costo, por lo tanto la presente revisión describe su tratamiento actual. Metodología de búsqueda: se realizó una búsqueda bibliográfica en las bases de datos Pubmed, Cochrane, LILACS, SciELO y el motor de búsqueda Google Scholar, seleccionándose 140 artículos para revisión de los cuales 48 artículos originales y 50 revisiones fueron referenciados. Discusión: como tratamiento en las personas de edad, se recomienda la ingesta de al menos 1,2 g/kg de proteína al día, acompañada de un aporte suficiente de micronutrientes y ocasionalmente de suplementos proteicos. Los programas de entrenamiento de fuerza en personas mayores es actualmente un método útil para la misma. El uso de fármacos debe ser evaluado individualmente. Conclusiones: la adecuada alimentación y el ejercicio de resistencia constituyen los pilares fundamentales del tratamiento y prevención de la sarcopenia. MÉD.UIS. 2017;30(2):71-81.

Palabras claves: Sarcopenia. Envejecimiento. Fuerza Muscular. Terapéutica.

\section{Generalities and treatment of Sarcopenia}

\section{ABSTRACT}

Introduction: the sarcopenia makes reference to the mass and muscular force's decrease related with the mechanisms of the aging and generates a functional commitment of high cost, therefore the present revision describes its current treatment. Methodology: a bibliographic search was carried out in Pubmed, Cochrane, LILACS, SciELO and Google Scholar databases, selecting 140 articles to be reviewed of which 48 original articles and 50 reviews were referenced. Discussion: as a treatment for sarcopenia in elderly population, it is recommended to take at least $1.2 \mathrm{~g} / \mathrm{kg}$ of protein per day, accompanied by a sufficient supply of micronutrients and occasional protein supplements. Strength training programs in older people is currently a useful method to combat this condition. The use of drugs should be evaluated individually. Conclusions: to prevent and treat sarcopenia, adequate nutrition and resistance exercise are the main pillars of treatment. MÉD.UIS. 2017;30(2):71-81.

Keywords: Sarcopenia. Aging. Muscular Strength. Therapy.

¿Cómo citar este artículo?: Hernández-Rodríguez J, Licea-Puig ME. Generalidades y Tratamiento de la Sarcopenia. MÉD.UIS. 2017;30(2):71-81.

\section{INTRODUCCIÓN}

La esperanza de vida en la población mundial ha aumentado en las últimas décadas', lo que ha conllevado a considerarse adulto mayor a aquella persona con 60 años o más, no obstante aún persiste la discusión en torno a esto. Lo anterior se fundamenta en que no todas las personas envejecen de la misma manera, por lo que se considera la existencia de un "tiempo de envejecimiento" propio

Artículo recibido en Noviembre 15 de 2016 y aceptado para publicación en Abril 05 de 2017 
para cada persona ${ }^{2}$. En Cuba, para el año 2000 los adultos mayores constituían el 12,9\% de la población general, cifra que se incrementó a $19 \%$ en el $2014^{3}$ con lo que se evidenció un aumento del envejecimiento poblacional y sus consecuencias, una de ellas la Sarcopenia (Sp).

Para definir el término $\mathrm{Sp}$, es necesario comprender el significado de la palabra fragilidad. Distintas definiciones han incluido aspectos físicos, cognitivos y psicosociales y la mayoría de ellas, concuerda en que es un estado relacionado al envejecimiento ${ }^{4}$. Fried ${ }^{5}$ definió esta última como la presencia de por lo menos tres de cinco criterios: fatiga crónica auto-referida, debilidad, inactividad, disminución de la velocidad de marcha y pérdida de peso. Este modelo fue replicado y sobre él se construyó uno moderno con identificación de factores causales fisiológicos, como la activación del sistema de inflamación y la alteración del sistema inmunitario, endocrino y músculo-esquelético ${ }^{4}$. Por otro lado, para Silva y colaboradores ${ }^{6}$ la Sp es una de las variables utilizadas para definir el síndrome de fragilidad, por lo que estos dos pueden coexistir y superponerse en un mismo anciano.

Existe controversia en considerar la Sp como una enfermedad, debido a que la pérdida de masa muscular esquelética se considera un fenómeno inherente al proceso de envejecimiento. Aunque, cuando esta es grave y causa dependencia funcional e interfiere negativamente en la calidad de vida de los ancianos, se califica como tal7. Para el Grupo de Trabajo Europeo sobre Sarcopenia en Ancianos ${ }^{8}$, la Sp es definida como "un síndrome caracterizado por una pérdida progresiva y generalizada de la masa y fuerza del músculo esquelético con riesgo de sufrir consecuencias adversas como: discapacidad física (disminución de la capacidad funcional del músculo), mala calidad de vida y muerte"; lo que coincide con el criterio de otros autores $^{9-11}$.

Diferentes estudios muestran variabilidad en la prevalencia de la Sp. Esto se debe a diferentes factores, principalmente a las características de la población estudiada y a la metodología empleada en su diagnóstico ${ }^{12}$. Según Morley ${ }^{13}$, la prevalencia en personas de 60-70 años es del 5-13\%, mientras que en los mayores de 80 años oscila entre el 1150\%. La Sp representa un deterioro del estado de salud y compromiso funcional de alto costo personal a causa de los trastornos del movimiento, que aumentan el riesgo de caídas y fracturas, el deterioro en la capacidad de realizar actividades cotidianas, la pérdida de la independencia y la probabilidad de muerte, conllevando a un mayor uso de los servicios de salud y por ende un incremento en el gasto socio sanitario de difícil solución ${ }^{14,15}$. Por esta razón, la presente revisión tiene como objetivo describir el tratamiento actual de la Sp.

\section{Metodología de Búsqueda}

Se obtuvieron un total de 148 artículos a través de las bases de datos Pubmed, Cochrane, LILACS, SciELO y el motor de búsqueda bibliográfica Google Scholar, de los cuales 140 fueron seleccionados para revisión. Se emplearon como términos de búsqueda "Sarcopenia”, “Envejecimiento”, "Fuerza Muscular y Terapéutica" y fueron utilizados los filtros idioma en español o inglés, artículos originales de investigación y de revisión y edad mayor o igual a 60 años. Fueron excluidos aquellos que no se ajustaban al objetivo de la revisión referenciándose finalmente 98 artículos, de los cuales 48 correspondían a artículos originales y 50 a revisiones.

\section{Fisiopatología}

Los mecanismos fisiopatológicos de la Sp no están claros y quizás intervengan factores musculares primarios y otros que no dependen directamente de él (secundarios). En este sentido se describen varios elementos en su etiología, entre los cuales se encuentran: la disfunción mitocondrial, el estrés oxidativo, los estados pro inflamatorios, la pérdida de neuronas motoras, las alteraciones de la placa neuromuscular o el desequilibrio entre la denervación y la re inervación; así como los cambios hormonales (en la insulina, andrógenos, estrógenos, hormona de crecimiento, factor de crecimiento similar a la insulina-1, Vitamina D (Vit D) y hormona paratiroidea, entre otros) producto del envejecimiento ${ }^{16}$. A estos elementos, se les pueden sumar las alteraciones producidas por enfermedades metabólicas o de otro tipo ${ }^{16}$, lo que posibilita el incremento de los procesos catabólicos que se dan en el músculo estriado y sus deletéreas consecuencias en términos de discapacidad y morbimortalidad ${ }^{17}$.

Un proceso importante que caracteriza a los músculos en el envejecimiento es la infiltración 
grasa. Esta se produce tanto a nivel macroscópico (entre los grupos musculares) como microscópico (entre los miocitos y su interior). Existen evidencias que correlacionan el porcentaje de masa grasa con la cantidad de deposición lipídica intramiocelular, la cual es utilizada como una medida aproximada de adiposidad $^{16}$. Para su determinación se ha propuesto métodos como la medida del porcentaje de grasa corporal a través de la absorciometría de rayos $\mathrm{X}$ de doble energía, el análisis de impedancia bioeléctrica, la tomografía axial computarizada o la resonancia magnética nuclear ${ }^{18,19}$.

La infiltración grasa a nivel muscular crea las bases para el desarrollo de la obesidad sarcopénica, para la cual todavía no existe una buena definición operativa, sin embargo consiste en la pérdida de masa magra en presencia de tejido adiposo normal o aumentado; esto puede contrarrestar el incremento del peso corporal causado por el aumento de la grasa, por lo que no siempre se asocia a un descenso del índice de masa corporal, constituyendo un importante riesgo para la salud ${ }^{6,10,20-22}$.

\section{Diagnóstico}

El diagnóstico de la Sp, se basa fundamentalmente en la medida de la masa y fuerza muscular, determinando puntos de corte que se correlacionan con el riesgo de aparición de complicaciones ${ }^{12}$, sin dejar a un lado la evaluación del rendimiento físico ${ }^{8}$. A pesar de que su diagnóstico no está totalmente estandarizado, se habla de Sp cuando la masa muscular es inferior a dos desviaciones estándar del valor promedio de la que presentan personas sanas, del mismo sexo y $\operatorname{edad}^{23}$.

Los métodos de evaluación de la composición corporal a través de las técnicas de imagen, permiten determinar la masa libre de grasa y la muscular esquelética ${ }^{12,14}$, aunque el desarrollo de las ecuaciones de predicción pueden estimar la masa muscular de forma indirecta ${ }^{14}$. Mientras, la fuerza muscular se puede estimar mediante un dinamómetro que mide la fuerza de prensión o agarre, o por medio de la fuerza de flexión y extensión de rodilla ${ }^{12,14}$.

La capacidad o rendimiento físico se recomienda evaluarlo a través del uso de distintas pruebas, cuyos resultados reflejan la habilidad que tiene el paciente para desarrollar actividades cotidianas asociadas a la pérdida de la masa muscular. Entre ellas se describen la velocidad de la marcha (caminar cierta distancia y medir con un cronómetro el tiempo) y la evaluación de los movimientos, donde el individuo cambia de posición sentado a de pie, camina hasta un punto y se devuelve y retorna a su posición inicial ${ }^{12,14}$.

La Sp tiene una serie de causas, por esta razón se clasifica en primaria o secundaria. De este modo, la primaria está relacionada con la edad, en ausencia de otros desencadenantes claves; la secundaria tiene como etiología el sedentarismo, la nutrición inadecuada o la enfermedad (falla orgánica avanzada, enfermedad inflamatoria, neoplasias), de ahí que también puede ser vista en adultos jóvenes ${ }^{8,24}$.

\section{Tratamiento de la Sarcopenia}

En la medida que se conoce más sobre la fisiología del tejido muscular y adiposo, se comprende mejor sus interacciones 25 . El desbalance entre factores anabólicos y catabólicos y el papel de la inflamación como mediador importante entre ambos procesos ${ }^{25}$ permite un mejor enfoque terapéutico (Ver Figura 1) en el paciente senil y así poder lograr una sociedad que garantice años de vida con calidad a este grupo poblacional ${ }^{26}$.

\section{Tratamiento Preventivo de la Sarcopenia}

La Sp presenta factores de riesgo no modificables relacionados con la constitución física del individuo, entre ellos ser mujer, la susceptibilidad genética y el proceso de envejecimiento; en contraste están aquellos modificables como el bajo peso al nacer, el estilo y condiciones de vida (fumar, inactividad física, malnutrición, etc.), así como muchas enfermedades que secundariamente pueden facilitar su presencia ${ }^{24}$ y sobre las cuales se puede actuar para prevenir su desarrollo.

Por otro lado, en la senectud el gusto y el olfato están disminuidos, la sensación de sed es menor y la saciedad es más precoz generando una tendencia a ingerir una menor cantidad de alimentos en comparación a adultos de otras edades ${ }^{33}$. A esto contribuye la presencia de problemas de salud, económicos, sociales u originados de una situación de dependencia funcional. Lo anterior, conlleva a la pérdida progresiva de peso a partir de los 65 años (250-500 g/año), acompañado de una disminución de la masa muscular de unos $3 \mathrm{~kg} /$ década, lo cual podría originar $\mathrm{Sp}^{33}$. Por lo tanto, la práctica de estilos de vida saludable se convierte en una medida preventiva en el desarrollo de la $\mathrm{Sp}^{32}$. 


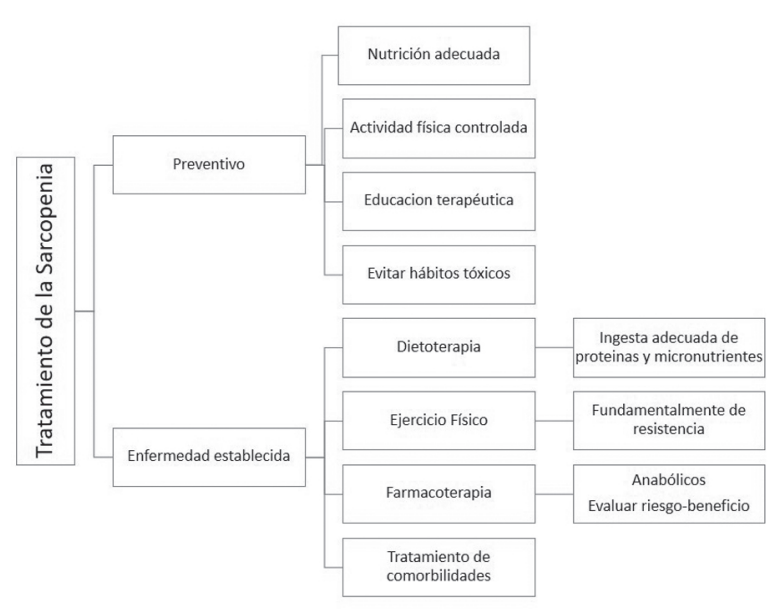

Figura 1. Tratamiento de la Sarcopenia

Fuente: Rondanell M, Perna S, Faliva MA, Peroni G, Infantino V, Pozzi R. Novel insights on intake of meat and prevention of sarcopenia: all reasons for an adequate consumption. Nutr hosp. 2015;32(5):2136-43.27

Padilla Colón CJ, Sánchez Collado P, Cuevas MJ. Beneficios del entrenamiento de fuerza para la prevención y tratamiento de la sarcopenia. Nutr Hosp. 2014;29(5):979-88.28

Del Rey Rozas T. Síndrome de Sarcopenia. Trabajo Fin de Grado Universidad de Cantabria [Internet]. 2014 [citado 29 Dic 2016]. Disponible en: https://repositorio.unican.es/xmlui/bitstream/ handle/10902/5204/ReyRozasT.pdf?sequence $=129$

Paladines B, Quizhpi M, Villota P. Tratamiento integral de la sarcopenia senil. Revista de la Facultad de Ciencias Químicas. 2016;3:41-8.30

Falque-Madrid L. La evidencia científica y el arte de envejecer. An Venez Nutr 2014;27(1):110-8. 31

Malafarina V, Uriz-Otano F, Gil-Guerrero L. Valoración y tratamiento nutricional de la sarcopenia. Rev Esp Geriatr Gerontol 2013;48(4):153-4. 32

Los ancianos constituyen una población capaz de recibir una intervención nutricional controlada, lo que permitiría mejorar la salud y reducir el riesgo de desarrollar deterioro funcional basado en ciertas medidas, entre las que se destaca la corrección de conductas dietéticas inadecuadas desde el punto de vista cuantitativo y cualitativo, asociado a la organización de los horarios de las comidas y la solución de los posibles factores que dificultan la ingesta, como la escasa higiene bucal o ausencia de dentadura 34,35 .

Se reporta que una nutrición adecuada retrasa la progresión de este síndrome y contrarresta la resistencia anabólica, es así como la ingesta de proteínas de alto valor biológico a lo largo de la vida y no haber sufrido malnutrición generan un menor impacto en la disminución de la masa muscular inherente al proceso de envejecimiento ${ }^{20}$. Se recomienda el consumo de carne (res, cordero, cerdo, pescado) y huevos ya que contienen compuestos biológicamente activos tales como la creatina, la carnitina y el ácido linoleico conjugado, que tienen impacto significativo sobre el metabolismo proteico humano ${ }^{30}$.

Otro elemento de importancia en la prevención de la Sp es la práctica frecuente de ejercicio físico ya que mejora la funcionalidad e independencia del anciano, su estado de ánimo y calidad de vida ${ }^{36,37}$. Lo anterior se evidencia en un estudio realizado por Ryu et al. ${ }^{8}$, en una población de adultos mayores coreanos donde concluyen que la actividad física alta o moderada es un factor protector para obesidad sarcopénica en hombres y mujeres $(\mathrm{OR}=0,27$ y $\mathrm{OR}=0,43$ para actividad física alta respectivamente); aunque este estudio no mostró una relación estadísticamente significativa entre la actividad física y $\mathrm{Sp}$ en el sexo femenino respecto al masculino, otros estudios indican que este beneficio se observa en ambos sexos y es proporcional al aumento en la intensidad del ejercicio físico, por lo que se recomienda realizar ejercicios aeróbicos de intensidad moderada durante 30 minutos, cinco días por semana, para obtener buenos resultados ${ }^{37}$.

El ejercicio aeróbico debe incluir un programa de al menos dos días no consecutivos por semana de ejercicios de resistencia, por lo que se recomienda practicar ocho a diez ejercicios que desarrollen la fuerza de la mayor parte de grupos musculares. Los programas de entrenamiento de fuerza o resistencia en personas mayores probablemente constituyen una de las medidas preventivas más eficaces para retrasar la aparición de la $\mathrm{Sp}^{27}$. Adicionalmente, se debe asociar ejercicios que mantengan la flexibilidad de los músculos y tendones, para esto se sugiere dos sesiones de 8-10 repeticiones con una duración de diez minutos ${ }^{37}$.

Mediante audiencias sanitarias, clases destinadas a los adultos mayores o consejos en consulta, se podría establecer la base de una intervención nutricional y de actividad física controlada para lograr la corrección del sedentarismo y conductas alimentarias inadecuadas, lo cual mejoraría la salud y favorecería un envejecimiento saludable y activo ${ }^{27,32}$.

Evitar hábitos tóxicos fundamentalmente el tabaco ${ }^{39,40}$ y el alcohol (sobre todo en personas con enfermedades hepáticas) ${ }^{41}$ resulta útil en la prevención de la Sp, así como el uso crónico y a dosis excesivas de algunos medicamentos, entre los que se encuentran los corticosteroides y las hormonas tiroideas, ya que tienen un papel catabólico sobre la masa muscular contribuyendo al desarrollo de la 
Sp en algunas personas ${ }^{12,42}$. Por esta misma razón se debe tratar eficazmente las enfermedades que favorezcan el desarrollo de la misma ${ }^{24}$.

Un aspecto más a tener en cuenta, es que los adultos mayores deben centrarse en el mantenimiento del peso corporal, pues su disminución puede dar lugar a una pérdida indeseable de masa muscular. Los obesos mórbidos que necesitan perder peso deben ser aconsejados para mantener la ingesta de proteínas y el ejercicio a un nivel capaz de preservar la masa muscular. La prevención de la Sp no sólo dará lugar a importantes ahorros en costos de salud, sino también contribuirá a una mejor salud en la senectud ${ }^{43}$.

\section{Tratamiento de la Sarcopenia ya establecida}

\section{DIETOTERAPIA}

Con el envejecimiento la ingesta calórica y proteica se reduce por diversos factores ${ }^{43,44}$. Existe evidencia que asocian la ingesta insuficiente de proteínas, de Vit $\mathrm{D}$, nutrientes antioxidantes y ácidos grasos poliinsaturados $\mathrm{n}_{3}$ de cadena larga a una mala función física ${ }^{45}$. La vitamina C, E, los carotenoides y los ácidos grasos poliinsaturados, actúan como antiinflamatorios, mientras otros nutrientes como los ácidos grasos saturados y los trans-monosaturados tienen efectos pro-inflamatorios ${ }^{46}$.

La ingesta adecuada de nutrientes que aporten energía y en particular proteínas, es un componente de utilidad en el tratamiento de la Sp. Los aminoácidos esenciales son necesarios para estimular el crecimiento muscular y la única forma de obtenerlos es por medio de la dieta ${ }^{47,48}$. De esta forma, es importante garantizar una correcta suplementación de proteínas de alto valor biológico que aportan una elevada concentración de aminoácidos esenciales y de Vit $D$, para mantener la masa muscular.

De hecho, se reporta que una ingesta proteica de al menos $0,8 \mathrm{~g} / \mathrm{Kg} /$ día, incrementaría los niveles del factor de crecimiento similar a la insulina-1, el cual tiene un efecto anabólico sobre el músculo49; adicionalmente esta medida podría aumentar la fuerza muscular ${ }^{47}$. Algunos autores ${ }^{49}$ sugieren consumir 1,2 g/ kg de proteína al día, para conservar la masa muscular y mantener el balance nitrogenado sin causar efectos negativos en la función renal de los adultos mayores. No obstante, otros investigadores ${ }^{50,51}$ recomiendan cantidades superiores (1,2-1,5 g/kg/día de proteínas) incluso para prevenirla.
Según Paddon et al.52, el envejecimiento no reduce la respuesta anabólica proteica de alta calidad y sugieren la ingesta de aproximadamente $25-30 \mathrm{~g}$ de proteína por comida (10-15g de aminoácidos esenciales), que contribuiría a estimular al máximo la síntesis de proteína muscular en los ancianos. Otro de los aspectos que se recomiendan es la distribución del consumo proteico durante el día, lo cual desempeña un papel fundamental en el mantenimiento de la masa y función muscular ${ }^{48,52}$. Los aminoácidos de cadena ramificada, especialmente la leucina (4g/comida, tres veces al día), son beneficiosos en personas con riesgo de $\mathrm{Sp}$, especialmente en inmovilizados o con enfermedades neurológicas ${ }^{51}$, ya que en esta última actúa como secretagogo y favorece la traducción y síntesis de proteína muscular, por eso se debe indicar el consumo de alimentos ricos en esta como la leche, queso, carne, atún, pollo, maní, soya, trigo y huevos ${ }^{53}$. Los pacientes que por algún motivo no puedan cumplir con esta última recomendación, pueden optar por suplementos proteicos ricos en leucina ${ }^{49,51}$.

La hipovitaminosis D puede producir Sp y debilidad muscular ${ }^{54}$. Se ha establecido la relación entre concentraciones bajas de Vit $\mathrm{D}$ como factor de riesgo para desarrollar $\mathrm{Sp}$ en ancianos que se expresa en forma de degradación miofibrilar, disminución del recambio proteico y de la secreción de insulina mediada por la hipocalcemia ${ }^{50,55}$. Esta vitamina se almacena en mayor proporción en los tejidos hepático, muscular y adiposo. Las personas que se exponen poco al sol, las de piel oscura, las que habitan en latitudes elevadas y los obesos tienen mayor probabilidad de sufrir su deficiencia ${ }^{56}$. La forma habitual de adquirirla es mediante la exposición directa de la piel a la luz solar, no obstante en el caso de los adultos mayores este mecanismo pierde eficacia y los riñones tienen menor capacidad de convertirla a su forma activa ${ }^{58}$. Se recomienda dosis profilácticas de $600 U \mathrm{Ul} /$ día en adultos de 19 a 70 años y de $800 U$ I/día a partir de los 71 años y cantidades entre 800-1000Ul/día en personas con Sp ${ }^{57,58}$.

Esta suplementación debe ser controlada a través de la determinación periódica de los niveles séricos de hidroxivitamina D (25OHD) 57,58 . En general, niveles de $50 \mathrm{nmol} / \mathrm{L}$ en suero cubriría las necesidades del $97,5 \%$ de la población. El límite inferior del rango de requerimientos es de $30 \mathrm{nmol} / \mathrm{L}$, pues los síntomas de deficiencia pueden aparecer a niveles inferiores a este dependiendo de diferentes factores ${ }^{57}$.

Existen alimentos que contienen Vit $D$ y de los cuales el adulto mayor puede beneficiarse, entre ellos 
el aceite de hígado de bacalao, pescados con un alto porcentaje de grasas (salmón, atún, caballa), carnes rojas y vísceras (hígado vacuno), huevos (la yema) ${ }^{57,59}$. El champiñón común contiene ergosterol, que se convierte en Vit $D$ cuando se expone a una cantidad adecuada de rayos solares y en la actualidad se estudia la forma de vender este producto enriquecido con la misma. Otra de sus ventajas es su bajo nivel calórico y su aporte de minerales como el selenio, cobre y potasio, que ayudan a que funcionen de mejor forma los músculos y los nervios ${ }^{60}$.

Está ampliamente demostrado que las ayudas ergogénicas nutricionales ofrecen beneficios fisiológicos directos para el rendimiento en el ejercicio y su recuperación. La suplementación con ß-hidroxiß-metilbutirato, un metabolito natural de la leucina, es un elemento de interés ya que puede disminuir el catabolismo del músculo ${ }^{61}$, aumenta la fuerza y masa muscular magra en sujetos no entrenados que comienzan a realizar ejercicio ${ }^{62}$. La dosis más eficaz descrita como suplemento es de 3 g al día51.

Otra ayuda ergogénica descrita es el mono hidrato de creatina. La creatina es un compuesto nitrogenado que se combina con fosfato originando la fosfocreatina, responsable de la resíntesis de trifosfato de adenosina mediante una reacción catalizada por la enzima creatinkinasa63. La anterior representa una fuente de energía almacenada extra mitocondrial y su incremento mejora la masa muscular y la fuerza flexora-extensora de la rodilla en los adultos mayores ${ }^{64}$, aunque su uso puede provocar retención de líquidos y por ende edema periférico en las personas de edad ${ }^{50}$.

\section{EJERCICIO FÍSICO}

La pérdida de masa magra es directamente proporcional a la cantidad de masa muscular e inversa al uso de la misma ${ }^{65}$. La estimulación muscular que se produce con el ejercicio, se relaciona con un mayor uso de los aminoácidos esenciales de la dieta sugiriendo que en las personas mayores el aumento de la actividad física tiene efectos protectores a nivel muscular $^{65}$.

Entre los beneficios del ejercicio se describen la prevención del deterioro cognitivo y las caídas ${ }^{63}$, disminución de la resistencia a la insulina a través de varios mecanismos, entre los que se señalan el restablecimiento de la cascada a la señal insulínica y el decremento del efecto deletéreo de los lípidos intramusculares que mejora la oxidación de dichas grasas y la contracción muscular mediada por la activación de 5' AMP-activated protein kinase, lo que conduce a la translocación del GLUT4 incrementando la comunicación muscular pancreática mediante mioquinas ${ }^{66}$.

Lo anterior indica la alta eficacia del ejercicio físico en la producción de mejoras cualitativas y cuantitativas a nivel del músculo esquelético ${ }^{67}$, por lo que se identifica como un método relativamente seguro, incluso en aquellos con múltiples comorbilidades ${ }^{68,69}$. No obstante Liu et al. ${ }^{70}$, sugieren tener cierta precaución con la implementación de programas de ejercicios de resistencia progresiva en poblaciones de ancianos con Sp, debido a posibles efectos adversos. Malafarina et al. ${ }^{71}$, recomiendan solo limitarlos en pacientes con insuficiencia cardíaca congestiva.

\section{EJERCICIO DE RESISTENCIA}

Consiste en utilizar la fuerza para lograr la contracción muscular e incremento de la resistencia anaeróbica, la fuerza y el tamaño muscular. Esto mejora las tareas que requieren una buena funcionalidad, tales como levantarse del suelo o subir escaleras ${ }^{63,72}$. Además, se reporta mejores resultados al combinar la suplementación proteínica con el ejercicio de resistencia, principalmente cuando se administra inmediatamente después del ejercicio. Sin embargo, el empleo de suplementos proteínicos sin ejercicio no ha demostrado eficacia en el aumento de la masa muscular ${ }^{35}$.

Los programas de entrenamiento de fuerza varían en su intensidad, número de series, frecuencia y duración de la actividad física según su objetivo ${ }^{63}$. Los ancianos con funcionalidad conservada, sin comorbilidades y con un nivel de actividad física previa elevado, necesitaran un tipo de entrenamiento más exigente y de mayor intensidad que aquellos sedentarios ${ }^{74}$. Estos pueden ser realizados en el hogar o gimnasio; se recomienda realizarlos tres veces por semana y ser supervisados inicialmente por personal capacitado ${ }^{71}$.

Dichos entrenamientos probablemente constituyen una de las medidas preventivas más eficaces para retrasar la aparición de Sp en los adultos mayores y es actualmente uno de los métodos más útiles para combatirla mediante la estimulación de la hipertrofia e incremento de la fuerza ${ }^{63,73}$. Una revisión de Arnold et al. ${ }^{74}$, apoya este criterio al afirmar que 
el ejercicio es significativamente superior a todas las intervenciones conocidas (farmacológicas, nutricionales y hormonales) para estabilizar y revertir la Sp. Por otro lado, aquellos programas que aumentan progresivamente su intensidad permiten obtener mejorías morfológicas en la población anciana ${ }^{67}$, aunque se ha señalado que para observar los efectos positivos del ejercicio de alta intensidad se requiere mínimo 10-12 semanas ${ }^{71}$.

\section{EJERCICIO AERÓBICO}

La actividad aeróbica ha sido el paradigma del ejercicio físico para la mayoría de personas que inician un programa de acondicionamiento a causa de los beneficios que otorga su práctica en la promoción de la salud, el bienestar y la estética ${ }^{75}$. Se define como ejercicio aeróbico todo aquel que se realice de forma repetitiva, prolongada y sin interrupción alguna. Este incrementa el número de capilares por fibra muscular en una sección transversal muscular determinada, mejorando la perfusión sanguínea muscular e incluye actividades como caminar, correr, marchar, remar, nadar, pedalear, esquiar, subir escaleras, entre otros ${ }^{75}$.

Se recomienda realizar dichos ejercicios con una baja intensidad y en lo posible con el mínimo impacto sobre las articulaciones ${ }^{76}$. Aunque posteriormente (si es bien tolerado) se puede practicar una marcha a un ritmo más intenso, acompañada con un balanceo enérgico de brazos que se llevan en semiflexión, y una amplitud de movimientos mayor al de la caminata normal. Autores como Cruz-Jentoft et al. ${ }^{11}$, han observado que los ejercicios aeróbicos de intensidad media o moderada, a un $60 \%$ de la frecuencia cardiaca máxima, mejoran las funciones cardiovasculares y desempeño en actividades tales como caminar o levantarse de una silla.

En la tercera edad, se recomienda la realización de alguna de estas actividades mínimo tres días a la semana, con una duración de 20-60 minutos por sesión a una intensidad del 40-60\% de la frecuencia cardiaca máxima. El aumento del tiempo y la intensidad debe ser progresivo ${ }^{13}$. No obstante, con su práctica no se ha observado una asociación positiva con la detención del proceso de la Sp, como sucede con el ejercicio de resistencia75.

\section{EJERCICIOS DE FLEXIBILIDAD Y EQUILIBRIO}

Estas clases de ejercicios en el adulto mayor aunque no mejoran la $\mathrm{Sp}$, contribuyen a que los músculos y los tejidos mantengan la estructura del cuerpo en su lugar. Un entrenamiento regular con el tiempo, puede ayudar a desarrollar la agilidad corporal y a la prevención de caídas y heridas77, por lo tanto pueden ser incluidos como complemento a las actividades físicas descritas anteriormente ${ }^{11,67,77}$, aportando un mayor bienestar y mejor pronóstico al paciente.

\section{FÁRMACOS PARA EL TRATAMIENTO DE LA SARCOPENIA}

Con la edad disminuyen una serie de hormonas que se relacionan directamente con las alteraciones observadas en diversos procesos fisiológicos ${ }^{11}$. La testosterona es un esteroide anabolizante que se une a los receptores androgénicos en la musculatura y otros órganos, estimulando la síntesis de proteínas, entre ellas la actina y la miosina (proteínas contráctiles productoras de energía y generadoras de fuerza muscular). Se describe que la administración de anabolizantes aumenta el tejido muscular y el contenido mineral óseo ${ }^{78}$. Esta hormona ha sido empleada como tratamiento sustitutivo en varones jóvenes con hipogonadismo, en los cuales se ha observado un incremento en la masa magra²3.

Según Wang et al.79, la testosterona a bajas dosis aumenta la masa muscular y en dosis más altas la fuerza muscular. Sin embargo, no produce cambios significativos en la composición corporal, ni en la reducción del riesgo de caídas y fracturas o mejoría en la calidad de vida ${ }^{80}$. Debido a esto y a su importante perfil de efectos adversos, no se aconseja su uso en varones ancianos, al menos hasta que no se disponga de estudios más rigurosos que apoyen lo contrario ${ }^{23}$.

Otros anabolizantes (dehidroepiandrosterona, oxandrolona, androstendiona) y los moduladores selectivos del receptor androgénico, están en fase de estudio, aunque conviene resaltar que hasta el momento carecen de los efectos secundarios de la testosterona, con la cual comparten su efecto anabolizante 23 . El Ostarine es un producto oral, que actúa de forma selectiva sobre estos receptores, y se ha observado que cuando es usado en adultos mayores sanos, se aumenta su masa muscular y capacidad para subir escaleras ${ }^{81}$.

La Hormona de Crecimiento Humano (HGH) es necesaria para el mantenimiento del músculo y del hueso, siendo deficiente en la población anciana, por lo que se hipotetiza que el tratamiento con $\mathrm{HGH}$ puede ser útil para tratar la $\mathrm{Sp}^{82}$. Papadakis et 
al. ${ }^{83}$, realizaron un ensayo aleatorizado controlado, doble ciego, en 52 hombres sanos mayores de 69 años de edad, con una capacidad funcional bien preservada, pero bajos niveles basales de factor de crecimiento insulínico tipo 1, a los que se administró $\mathrm{HGH}(0,03 \mathrm{mg} / \mathrm{kg}$ de peso corporal) o placebo tres veces a la semana durante seis meses, con el objetivo de determinar si el reemplazo de la $\mathrm{HGH}$ en hombres mayores mejoraba la capacidad funcional. En los pacientes tratados se observó un aumento en la masa de tejido magro con disminución del tejido adiposo, mejorando la composición corporal mas no la capacidad funcional, lo que coincide con lo referenciado por otros investigadores ${ }^{84,85}$. Sin embargo, Rudman et al. ${ }^{86}$, reportan en un estudio realizado por ellos que el tratamiento con $\mathrm{HGH}$ no incrementó la masa ni la fuerza muscular.

Se han realizado estudios combinando el efecto del ejercicio físico y la administración de $\mathrm{HGH}$, donde esta hormona no ha demostrado un efecto beneficioso adicional al conseguido con el ejercicio ${ }^{84,85}$. El tratamiento combinado de testosterona y $\mathrm{HGH}$, incrementa la masa corporal muscular en varones con hipogonadismo o con deficiencia de $\mathrm{HGH}$, pero estos resultados no van acompañados de un mejoramiento funcional, en tanto sus efectos colaterales se pueden evidenciar ${ }^{87-89}$.

La terapia a corto y largo plazo con estrógenos en mujeres posmenopáusicas tiene resultados inconstantes en relación con la masa muscular ${ }^{84}$. La terapia sustitutiva con estrógenos estaría justificada a partir de los datos que indican que las mujeres con osteoporosis y sarcopenia es un grupo de alto riesgo para presentar fracturas y discapacidad. Los estudios existentes actualmente no muestran ninguna modificación de la masa ni función muscular ${ }^{22}$. Como indica un estudio de Kenny et al. $9^{\circ}$, la Sp es tan común en las mujeres no obesas con terapia hormonal de remplazo con estrógenos a largo plazo como en aquellas que no la usan, lo que sugiere que esta no protege contra la pérdida muscular del envejecimiento.

Dentro de las mioquinas, la miostatina es una de las moléculas secretadas por las células musculares esqueléticas durante el ejercicio. Posee propiedades antiinflamatorias y actúa facilitando la disminución de la resistencia a la insulina ${ }^{91}$. Se ha desarrollado una proteína terapéutica análoga de la miostatina (ACE083) cuyo efecto se produce a través de la activación del receptor de activina II. Se encuentra en fase de investigación, y fue diseñada para aumentar la masa y fuerza muscular en los músculos y grupos musculares específicos. Se administra localmente y su efecto es dosis dependiente ${ }^{92,93}$, facilitando una disminución de la grasa y un incremento en el anabolismo óseo con mejoría de la densidad mineral ósea ${ }^{50}$.

El uso de los productos señalados anteriormente, debe ser evaluado de forma individual y sopesando el factor riesgo-beneficio, ya que hasta el momento no existe una terapia farmacológica realmente efectiva y exenta de efectos colaterales, que en algunos casos pudieran ser hasta contraproducentes, al ser aplicadas en el anciano con Sp.

\section{Tratamiento de Comorbilidades}

La Sp se puede producir como consecuencia de múltiples enfermedades y condiciones crónicas, lo que en ocasiones reduce las opciones de tratamiento y los resultados clínicos positivos. La mala nutrición y el sedentarismo, hacen que un número importante de individuos enfrenten el envejecimiento natural, con una masa muscular estructural y funcionalmente deteriorada ${ }^{94}$.

La elevada tasa de crecimiento de la obesidad y Diabetes Mellitus tipo 2 a nivel mundial, puso en evidencia la existencia de un estado inflamatorio crónico de baja intensidad -en ambas entidades- y su conexión con la pérdida de masa muscular desde edades tempranas, sobrecargando el músculo con triglicéridos y alterando su recambio proteico ${ }^{94}$.

En la Diabetes Mellitus tipo 2 esta aumentada la resistencia a la acción de la insulina que provoca una menor estimulación del anabolismo proteico y un aumento de su catabolismo, conllevando a la pérdida de la masa muscular ${ }^{16}$. Partiendo de lo anterior, se infiere que quizás la indicación de insulinosensibilizadores como la metformina reduciría la obesidad visceral, los niveles de factores pro inflamatorios y protrombóticos, garantizando un buen control metabólico. Esta medida terapéutica puede jugar un papel en la prevención y tratamiento de la Sp en el paciente senil con Diabetes Mellitus tipo 2 y obesidad ${ }^{66,95}$.

El tratamiento y control oportuno de las enfermedades donde subyace un estado pro inflamatorio crónico, y en general donde aumenta el catabolismo muscular, como la insuficiencia cardíaca, respiratoria, renal, la 
malnutrición proteico calórica, el hipercortisolismo endógeno y exógeno, los estados de tirotoxicosis, el cancer ${ }^{8,96-98}$, entre otros, es elemental en la prevención y tratamiento de la sarcopenia secundaria.

\section{CONCLUSIONES}

El tratamiento de la sarcopenia se basa en una serie de medidas que incluyen la dietoterapia, el ejercicio físico, la farmacoterapia y el tratamiento de los problemas de salud que concomitan con la SP. Las dos medidas principales para prevenir y combatir la pérdida de masa muscular son el ejercicio de resistencia y una adecuada alimentación con un aporte suficiente de macro y micronutrientes, donde el suministro adecuado de proteínas, vitamina $D$ y A, juega un papel fundamental. En este sentido, la educación terapéutica y el adecuado tratamiento de las comorbilidades son elementales.

Aunque el uso de fármacos no ha demostrado ser la solución, la testosterona a dosis bajas y altas aumenta la masa y fuerza muscular respectivamente. Sin embargo, debido a su perfil de efectos adversos, no se aconseja su uso en varones ancianos. Igualmente, se debe continuar investigando el posible uso de otros anabolizantes, que sean capaces de mejorar la SP con un mínimo de efectos adversos.

\section{Conflicto de InTereses}

Los autores declaran no tener conflicto de intereses.

\section{RefERENCIAS BibLIOGRÁFICAS}

1. Ward SA, Parikh S, Workman B. Health perspectives: international epidemiology of ageing. Best Pract Res Clin Anaesthesiol. 2011; 25(3):305-317.

2. Fernández IE, Martínez AJ, Santos CM. La salud y el envejecimiento poblacional. Memorias Convención Internacional de Salud Pública. Cuba Salud 2012. [Internet] 2012 [citado 4 Enero 2016]. Disponible en: http://www.convencionsalud2012.sld.cu/index. php/convencionsalud/2012/paper/viewFile/2044/820

3. MINSAP. Anuario estadístico de salud 2014. Ciudad de la Habana: Dirección Nacional de Registros Médicos y Estadísticas de Salud. MINSAP. La Habana. [Internet] 2015 [Accedido: 2016 Dic 26]; Disponible en: http://files.sld.cu/bvscuba/files/2015/04/ anuario-estadistico-de-salud-2014.pdf

4. Jauregui JR, Rubin RK. Fragilidad en el adulto mayor. Rev. Hosp. Ital. B.Aires.2012; 32(3):110-115.

5. Fried LP, Tangen CM, Walston J, Newman AB, Hirsch C, Gottdiener $\mathrm{J}$, et al. Frailty in older adults evidence for a phenotype. J Gerontol A Biol Sci Med Sci. 2001; 56(3): 146-157.

6. Silva TAA, Frisoli Junior A, Pinheiro MM, Szejnfeld VL. Sarcopenia associada ao envelhecimento: aspectos etiológicos e opções terapêuticas. Rev Bras Reumatol. 2006; 46(6): 391-397.

7. De Araújo LE, de Lima T, Nogueira GM, Mânica IB, Schneider RH, Valle MG. Envelhecimento, estresse oxidativo e sarcopenia: uma abordagem sistêmica. Rev. bras. geriatr. gerontol. 2012; 15(2): 365-380
8. Cruz-Jentoft A, Baeyens JP, Bauer JM, Cederholm T, Land F, Martin FC, et al. Sarcopenia: European consensus on de $\square$ nition and diagnosis. Report of the European Working Group on Sarcopenia in Older People. Age Agenig. 2010; 39(4): 412-423.

9. Chen LK, Liu LK, Woo J, Assantachai P, Auyeung TW, Bahyah KS, et al. Sarcopenia in Asia: Consensus Report of the Asian Working Group for Sarcopenia (AWGS). J Am Med Dir Assoc. 2014; 15(2): 95-101.

10. Evans WJ. Sarcopenia Should Reflect the Contribution of AgeAssociated Changes in Skeletal Muscle to Risk of Morbidity and Mortality in Elderly People. J Am Med Dir Assoc. 2015; 16(7):546547.

11. Cruz-Jentoft AJ, Triana FC, Gómez MC, López A, Masanés F, Martín PM, et al. La eclosión de la sarcopenia: Informe preliminar del Observatorio de la Sarcopenia de la Sociedad Española de Geriatría y Gerontología. Rev Esp Geriatr Gerontol. 2011; 46(2): 100-110.

12. Masané F, Navarro M, Sacanella E, López A. ¿Qué es la sarcopenia?. Semin Fund Esp Reumatol. 2010; 11(1):14-23.

13. Morley JE. Sarcopenia: diagnosis and treatment. J Nutr Health Aging. 2008; 12(7):452-456.

14. Ocampo JM, Reyes CA. Revisión sistemática de literatura. Declinación funcional en ancianos hospitalizados. Rev. Méd. Risaralda 2016; 22(1):49-57.

15. Sánchez J. Emponderamiento en torno al mayor ante el conflicto económico del desequilibrio demográfico. Atalaya Médica Turolense. 2016; X(6):73-79.

16. Rastogi R, Corriere M, Ferrucci L. Pérdida de masa muscular relacionada con la edad y enfermedades. Intra Méd. [Internet] 2014 [citado 6 Enero 2015]; 5(3). Disponible en: http://www. intramed.net/contenidover.asp?contenidoID $=83350$

17. Han HQ, Mitch WE. Targeting the myostatin signaling pathway to treat muscle-wasting diseases. Curr Opin Support Palliat Care. 2011; 5(4): 334-341.

18. Baumgartner RN, Koehler KM, Gallagher D, Romero L, Heymsfield SB, Ross R R, et al. Epidemiology of sarcopenia among the elderly in New Mexico. Am J Epidemiol. 1998; 147(8):755-763.

19. Schaap LA, Koster A, Visser M. Adiposity, muscle mass, and muscle strength in relation to functional decline in older persons. Epidemiol Rev. 2013; 35(1):51-65.

20. Hernández J, Licea M, Castelo L. Algunos aspectos de interés relacionados con la obesidad sarcopénica. Rev Cubana Endocrinol [Internet]. 2015 [Accedido: 2016 Feb 09]; 26(3): Disponible en: http://scieloprueba.sld.cu/scielo.php?script=sci arttext\&pid $=$ S1561-29532015000300007\&lng $=$ es.

21. Gómez AE, Rodríguez V, Vila S, Casajús JA, Ara I.Envejecimiento y composición corporal; la obesidad sarcopénica en españa. Nutr Hosp. 2012; 27(1):22-30

22. Walston JD. Sarcopenia in older adults. Curr Opin Rheumatol. November 2012;24(6):623-627.

23. Gómez AE. Sarcopenia. Ámbito farmacéutico. [Internet] 2011 [Accedido: 2016 Dic 29];30(4):. Disponible en: http:// apps.elsevier.es/watermark/ctl_servlet? f $=10 \&$ pident articulo $=90024752 \&$ pident_usuario $=0$ \& pident_revista $=4 \&$ fiche $_{-}$ $\mathrm{ro}=4 \mathrm{v} 30 \mathrm{n} 04 \mathrm{a} 90024752 \mathrm{pdf001}$.pdf\&ty $=92 \&$ accion $=$ L\&origen $=\mathrm{d}$ farmacia\&web $=w w w . d f a r m a c i a . c o m \& l a n=e s$

24. Cruz-Jentoft AJ. Relevancia clínica de la pérdida de masa muscular. Nutr Hosp. 2011; 4(1):3-6.

25. Zuñiga R. Barrientos $M$ Conceptos básicos sobre obesidad sarcopénica en el adulto mayor. Revista Clínica Escuela de Medicina UCR-HSJD. [Internet] 2015 [Accedido: 2015 Enero 6]; 5(3):7pag. Disponible en: https://scholar.google.com.cu/scholar?h $\mathrm{l}=\mathrm{es} \& \mathrm{q}=$ Conceptos $+\mathrm{b} \% \mathrm{C} 3 \% \mathrm{~A} 1$ sicos + sobre + obesidad + sarcop $\%$ C3\%A9nica + en + el +adulto + mayor.\&btnG $=\& l r=$

26. Berrío MI. Envejecimiento de la población: un reto para la salud pública. Rev Col Anest 2012; 40(3):192-194.

27. Rondanelli M, Perna S, Faliva M, Peroni G, Infantino V, Pozzi R. Novel insights on intake of meat and prevention of sarcopenia: all reasons for an adequate consumption. Nutr hosp. 2015;32(5):2136-43.

28. Padilla CJ, Sánchez Collado P, Cuevas MJ. Beneficios de entrenamiento de fuerza para la prevención y tratamiento de la sarcopenia. Nutr Hosp. 2014;29(5):979-88.

29. Del Rey Rozas T. Síndrome de Sarcopenia. [Internet]. 2014 [citado 29 Dic 2016]. Disponible en: https://repositorio.unican.es/xmlui/ bitstream/handle/10902/5204/ReyRozasT.pdf?sequence =1 
30. Paladines B, Quizhpi M, Villota P. Tratamiento integral de la sarcopenia senil. Revista de la Facultad de Ciencias Químicas. 2016;3:41-8.

31. Falque-Madrid L. La evidencia científica y el arte de envejecer. An Venez Nutr 2014;27(1):110-8.

32. Malafarina V, Uriz-Otano F, Gil-Guerrero L. Valoración y tratamiento nutricional de la sarcopenia. Rev Esp Geriatr Gerontol 2013;48(4):153-4.

33. alaba trueba.-, j., enrique arriola manchola., e., beobide telleria.-, i., calvo aguirre, j., muñoz diaz, j. and umerez urbieta, g. (2012). guía farmacoterapéutica para los pacientes geriátricos. 1st ed. vitoria-gasteiz: eusko jaurlaritza. osasun eta kontsumo saila $=$ gobierno vasco. departamento de sanidad y consumo

34. Valenzuela T. Efficacy of progressive resistance training interventions in older adults in nursing homes: a systematic review. Journal of American Medical Directors Association. 2012; 13(5):418-428.

35. Laviano A, Gori C, Rianda S. Sarcopenia and Nutrition (Review). Adv Food Nutr Res. 2014; (71):101-136.

36. Ortiz PA, Morales M. La importancia del ejercicio físico para el tratamiento de la Sarcopenia. Diss. [Internet] 2014 [Accedido: 2016 Dic 28]. Disponible en: http://bibliotecadigital.univalle.edu. co/bitstream/10893/6835/1/CD-0430813.pdf

37. Crespo - Salgado JJ, Blanco - Moure A. Prescripción de ejercicio físico: ¿cómo mejorar la adherencia? Medicina Clínica, 2012; 139(14):648-649.

38. Ryu M, Jo J, Lee Y, Chung YS, Kim KM, Baek WC. Association of physical activity with sarcopenia and sarcopenic obesity in community-dwelling older adults: the Fourth Korea National Health and Nutrition Examination Survey. Age and ageing 42(6); 734-740

39. Freitas ER, Araujo EC, Alves K. Influência do tabagismo na força muscular respiratória em idosos. Fisioter. Pesqui. 2012;19(4):32631.

40. Rom O, Kaisari S, Aizenbud D, Reznick AZ. Sarcopenia and smoking: a possible cellular model of cigarette smoke effects on muscle protein breakdown. Ann N Y Acad Sci. 2012;1259(1):4753.

41. Song DS, Chang UI, Choi S, Jung YD, Han K, Ko SH, et al. Heavy Alcohol Consumption with Alcoholic Liver Disease Accelerates Sarcopenia in Elderly Korean Males: The Korean National Health and Nutrition Examination Survey 2008-2010. PLoS One. 2016;11(9):e0163222.

42. Carceller DB, París AS, Oriz ES, López RGF, Calmarza P, Cortes VB, et al. Treatment of subclinical hyperthyroidism: effect on body composition. Nutr Hosp. 2015;32(5):2331-7.

43. Visvanathan R, Chapman I. Preventing sarcopaenia in older people. Maturitas. 2010;66(4):383-8.

44. Ferrando AA, Paddon-Jones D, Hays NP, Kortebein P, Ronsen O, Williams RH, et al. EAA supplementation to increase nitrogen intake improves muscle function during bed rest in the elderly. Clin Nutr. 2010;29(1):18-23.

45. Robinson S, Cooper C, Aihie Sayer A. Nutrition and sarcopenia: a review of the evidence and implications for preventive strategies. J Aging Res. 2012;2012:1-6.

46. García MN, Pons HE. Dieta e inflamación. An Venez Nutr. 2014;27(1):47-56.

47. Morley JE, Argiles JM, Evans WJ, Bhasin S, Cella D, Deutz NE, et al. Nutritional recommendations for the management of sarcopenia. J Am Med Dir AssoC. 2010;11(6):391-6.

48. Stout JR. Intervenciones nutricionales en sarcopenia. Nutr. hosp. 2011;4(1):7-8.

49. Cuesta F. Nutrición y sarcopenia: recomendación de ingesta proteica [Internet]. 2011 [Accedido: 2015 Enero 6]; Disponible en: http://www.sarcopenia.es/curso4_7.php

50. Morley JE. Sarcopenia in the elderly. Fam Pract. 2012;29(Suppl1):i44-i48.

51. Velázquez MC, Irigoyen ME, Delgadillo J. Salud muscular y prevención de sarcopenia: el efecto de la proteína, leucina y ß-hidroxi-ß-metilbutirato. Rev Metab Óseo y Min. 2012;10(2):98102.

52. Paddon-Jones D, Rasmussen BB. Dietary protein recommendations and the prevention of sarcopenia. Curr Opin Clin Nutr Metab Care. 2009;12(1):86-90.

53. Millward DJ, Fereday A, Gibson NR, Cox MC, Pacy PJ. Efficiency of utilization of wheat and milk protein in healthy adults and apparent lysine requirements determined by a single-meal [113C] leucine balance protocol. Am J Clin Nutr. 2002;76(6):132634.

54. Wolff A, Jones A, Hansen K. Vitamin D and Musculoske letal Health. Nat Clin Pract Rheumatol. 2008;4(11):580-8.

55. Sieber CC. Sarcopenia (Review). Ther Umsch. 2014;7(3):171-6.

56. Sánchez A, Oliveri B, Mansur JL, Fradinger E, Mastaglia S. Diagnóstico, prevención y tratamiento de la hipovitaminosis D. Rev. argent. endocrinol. metab. 2013;50(2):141-56.

57. Ross A, Taylor C, Yaktine A, Valle H. Dietary reference intakes for calcium and Vitamin D. 1st ed. Washington: National Academies Press; 2011.

58. Nutri-facts. Vit D: Recomendaciones para el consumo. [Internet] 2011 [Accedido: 2015 Enero 6]; Disponible en: http://www.nutrifacts.org/esp/vitaminas/vitamina-d-calciferol/recomendacionespara-el-consumo/

59. Apaza JJ. Vitaminas liposolubles. Rev Act Clin. 2014;41:2151-55

60. Melena Merchan. Los hongos, nueva fuente de vitamina D. Blog de la Sociedad Micológica de Gran Canaria. [Internet] 2008 [Citado 26 Feb 2017]; Disponible en: http:// sociedadmicologicadegrancanaria.blogspot.com/2008/03/loshongos-nueva-fuente-de-vitamina-d.html

61. Wilson JM, Lowery RP, Joy JM, Walters JA, Baier SM, Fuller JC $\mathrm{JR}$, et al. $\beta$-Hidroxy- $\beta$-methylbutyrate free acid reduces markers of exercise-induced muscle damage and improves recovery in resistance -trained men. Br J Nutr. 2013 Aug; 110(3):538-582.

62. Castell LM, Burke LM, Estear SJ, Maughan RJ. BJSM reviews: A-Z of nutritional supplements: dietary supplements, sports nutrition foods and ergogenic aids for health and performance Part 8. Br J Sports Med 2010; 44(6):468-70.

63. Padilla Carlos J. Efectos de un entrenamiento de fuerza y de la utilización de creatina en la prevención de la sarcopenia en personas de edad avanzada. Universidad de León. [Internet] 2014 [Citado 15 Feb 2016]; Disponible en: h https:/buleria.unileon.es/ bitstream/handle/10612/3602/tesis_0d90fe.PDF?sequence $=1$

64. Brose A, Parise G, Tarnopolsky MA. Creatine supplementation enhances isometric strength and body composition improvements following strength exercise training in older adults. J Gerontol A Biol Sci Med Sci. 2003;58(1):11-9.

65. Morley JE, Abbatecola AM, Argiles JM, Baracos V, Bauer J, Bhasin Sh, et al. Sarcopenia with limited mobility: An International Consensus. J Am Med Dir Assoc. 2011;12(6):403-9.

66. Egan B, Zierath J. Exercise metabolism and the molecular regulation of skeletal muscle adaptation. Cell Metab. 2013;17(2):162-84.

67. Mata F, Chulvi I, Heredia JR, Moral S, Marcos JF, Da Silva ME. Sarcopenia and resistance training: actual evidence. Journal of Sport and Health Research. 2013;5(1):7-24.

68. Gillespie LD, Robertson MC, Gillespie WJ, Lamb SE, Gates S, Cumming RG, et al. Interventions for preventing falls in older people living in the community. Cochrane Database Syst Rev. [Internet] 2009 [Citado 15 Feb 2016]; Disponible en: http:// onlinelibrary.wiley.com/doi/10.1002/14651858.CD007146.pub2/ full

69. García-Unciti M, Martínez JA, Izquierdo M, Gorostiaga EM, Grijalba A, Ibáñez J. Effect of resistance training and hypocaloric diets with different protein content on body composition and lipid profile in hypercholesterolemic obese women. Nutr Hosp. 2012; 27(5):1511-20.

70. Chiung-ju Liu, Nancy K Latham. Progressive resistance strength training for improving physical function in older adults. Cochrane Database Syst Rev. [Internet] 2009 [Citado 5 Ene 2016]; Disponible en: https://www.ncbi.nlm.nih.gov/pmc/articles/ PMC4324332/

71. V Malafarina, F Uriz-Otano, R Iniesta, Gil-Guerrero L. Sarcopenia in the elderly: diagnosis, physiopathology and treatment. Maturitas. 2012;71(2):109-14.

72. Chandler JM, Duncan PW, Kochersberger G, Studenski S. Is lower extremity strength gain associated with improvement in physical performance and disability in frail, community-dwelling elders?. Archives of physical medicine and rehabilitation. 1998; 79(1):2430.

73. Rolland Y, Dupuy C, Abellan van Kan G, Gillette S, Vellas B. Treatment Strategies for sarcopenia and frailty. Med Clin North Am. 2011;95(3): 427-38.

74. Arnold AS, Egger A, Handschin C. PGC-1a and myokines in the 
aging muscle - a mini-review. Gerontology 2011;57(1):37-43.

75. Realpe LE. Tendencia actual del tratamiento de la sarcopenia en el adulto mayor. Pontificia Universidad, Javeriana, Bogota DC. [Internet] 2014 [Citado 5 Ene 2016]; Disponible en: http:// repository.javeriana.edu.co/handle/10554/16070

76. Lioi L. Ejercicios aeróbicos para la tercera edad. Innatia. [internet] 2011 [Citado 4 Feb 2016]; Disponible: http://www.innatia.com/s/ c-ejercicio-tercera-edad/a-aerobicos-tercera-edad.html

77. BD. México. Ejercicio para adultos mayores con diabetes. [Internet] 2014 [citado el 29 de Abril de 2016]. Disponible en: http://www. bd.com $/ \mathrm{mexico} /$ diabetes $/$ main.aspx?cat $=3258 \& \mathrm{id}=31334$

78. Auriema AE, Ferrari D. Uso Terapêutico do Deca-Durabolin no Desmame da Ventilação Mecânica. portaldafisioterapia.com. br. [Internet] 2015 [citado el 29 de Abril de 2016]; Disponible en: http://portaldafisioterapia.com.br/uso-terapeutico-do-decadurabolin-no-desmame-da-ventilacao-mecanica/

79. Wang C, Nieschlag E, Swerdloff R, Behre HM, Hellstrom WJ, Gooren LJ, et al. Investigation, treatment and monitoring of lateonset hypogonadism in males. Eur J Endocrinol. 2008; 159(5): 507-514.

80. Gutiérrez LM, Ley S. Sarcopenia, diagnóstico diferencial e implicaciones terapéuticas. En: d'Hyver C, Gutiérrez LM. 3era edición. Geriatría. Méjico, DF. Editorial El Manual Moderno, 2014. p. 721-725.

81. García C. Enfermedades endocrinas en el adulto mayor. Rev Méd Clín Condes. 2013; 24(5):866-873.

82. Burgos R. Enfoque terapéutico global de la sarcopenia. Nutr. Hosp. 2006; 21(Suppl 3):51-60.

83. Papadakis MA, Grady D, Black D, Tierney MJ, Gooding GA, Schambelan M, et al. Growth hormone replacement in healthy older men improves body composition but not functional ability. Ann Intern Med. 1996;124(8):708-716.

84. Taaffe DR, Pruitt L, Reim J, Hintz RL, Butterfield G, Hoffman $\mathrm{AR}$, et al. Effect of recombinant human growth hormoneon the muscle strength response to resistance exercise in elderly men. J Clin Endocrinol Metab. 1994;79(5):1361-1366.

85. Yarasheski KE, Zachwieja JJ, Campbell JA, Bier DM. Effect of growth hormone and resistance exercise on muscle growth and strength in older men. Am J Physiol. 1995; 268(2):E268-276.

86. Rudman D, Feller AG, Nagraj HS, Gergans GA, Lalitha PY, Goldberg AF, et al. Effects of human growth hormone in men over
60 years old. N Engl J Med. 1990; 323(1):1-6.

87. Burgos R. Estrategia terapéutica para la desnutrición y la sarcopenia. Nestlé Nutrition Institute Workshop. [Internet] 2011 [citado el 30 de Diciembre 2016]. Vol(72)p.27. Disponible en: http://geriatricarea.com/estrategia-terapeutica-para-ladesnutricion-y-la-sarcopenia/

88. Cano CP. Sarcopenia. Rev. Act. Clin. Med. 2012; 17:824-828

89. Lynch GS, Schertzer JD, Ryall JG. Therapeutic approaches for muscle wasting disorders. Pharmacol Ther. 2007; 113(3):461-487.

90. Kenny AM, Dawson L, Kleppinger A, Iannuzzi-Sucich, Judge JO. Prevalence of sarcopenia and predictors of skeletal muscle mass in non-obese women who are long-term users of strogen replacement therapy. J Gerontol A Biol Sci Med Sci. 2003; 58(5):M436-40

91. Tencio JA, Alpizar D, Camacho S, Muñoz JP, Morales G. Mioquinas: mediadoras de los efectos del ejercicio físico en la salud. Revista Médica de la Universidad de Costa Rica. 2016; 10(2):32-43.

92. Morley JE. Wieght loss in older presons: new therapeutic approaches. Curr Pharm Des. 2007;13(35):3637-3647.

93. Acceleron. ACE-083 for muscle Loss. [Internet] 2016 [ citado: 15 de Febrero 2016]; Disponible en: http://www.acceleronpharma. com/products/ace-083/

94. Lanari A. Sarcopenia: repercusiones mecánicas y funcionales Rev Asoc Argent Traumatol Deporte. 2012;19(1):23-31.

95. Madiraju AK, Erion DM, Rahimi Y, Zhang XM, Braddock DT, Albright R, et al. Metformin suppresses gluconeogenesis by inhibiting mitochondrial glycerophosphate dehydrogenase. Nature. 2014; 510 (7506):542-546

96. Fappi A. Efeitos do ácido graxo ômega-3 na prevenção da atrofia muscular induzida pela dexametasona [dissertation]. São Paulo: University of São Paulo, Faculdade de Medicina; [Internet] 2013 [Accedido: 2016 Mayo 3]. Disponible en: http://www.teses.usp.br/ teses/disponiveis/5/5138/tde-13012014-114428/.

97. Altamirano M, Geovanny E. Desnutrición Intrahospitalaria: ELAN-Ecuador. Hospital Sagrado Corazón de Jesús, Área N. 2 Quevedo, Provincia de Los Ríos, 2011. [Internet] 2013 [Accedido: 2016 Mayo 3]. Disponible en: http://dspace.espoch.edu.ec/bitstre am/123456789/3968/1/20T00524.pdf

98. Valenzuela K, Rojas P, Basfifer K. Evaluación nutricional del paciente con cáncer. Nutr. Hosp. 2012;27(2):516-23. 